\title{
Acoustic transmission properties of pressurised and pre-stressed composite structures
}

\author{
T. Ampatzidis*a, Dr. D. Chronopoulos ${ }^{\mathrm{a}}$ \\ ${ }^{a}$ Institute for Aerospace Technology \& The Composites Group, The University of \\ Nottingham, NG7 2RD, UK
}

\begin{abstract}
This work was focused on the examination of the effect of the pre-stress, namely tension and pressure, on the wave propagation and acoustic behaviour of composite laminates. The dispersion characteristics of two dimensional layered and sandwich structures were predicted using Wave Finite Element Method (WFEM). The structures were examined in non-stressed and pre-stressed scenarios. After extracting the mass and stiffness matrix of a small periodic segment of the structure using commercially available Finite Elements software, a polynomial eigenvalue problem was formed, the solutions of which consisted of the propagation constants of the waves of the structure. This way the wavenumbers and eigenvectors of the out of plane structural displacements were extracted. These wave propagation magnitudes were then used to calculate important Statistical Energy Analysis (SEA) quantities, such as modal density and radiation efficiency. The effect of pre-stress on these quantities, along with its effect on loss factor of the structure were examined.

Keywords: Wave Finite Element, loss factor, Pre-stress, Sound Transmission

Loss
\end{abstract}

\section{Introduction}

Current research in most industries, such as aerospace and automotive, focuses on materials that offer low density along with superior dynamic and static

Email address: emxta3@nottingham.ac.uk (T. Ampatzidis*) 
performance. This goal has led to increasing use of sandwich structures and composite materials in general, whose high stiffness-to-weight ratio along with the tailoring of their properties that they offer make them quite appealing. This high stiffness-to-weight ratio they offer, though, comes with a significant cost in their vibroacoustical behaviour, being responsible for high noise and displacement resonant vibrations. Prompted by that, elevated quality and quantity of research is about modelling the behaviour of these materials, along with conventional ones, using time and cost efficient computational methods. These methods are used to reach the goal of enhanced stiffness, weight and vibration behaviour.

Classical publications $[1,2]$ offer analytic formulas to predict the wave propagation characteristics of numerous different structures. Classical Laminate Plate Theory (CLPT) is one of them [3], being developed as an extension of the Kirchhoff-Love's theory for isotropic panels and can be applied on thin orthotropic plates. Additionally, First-order Shear Deformation Theory (FSDT) [4] is based on the transverse shear deformation of the panel and can be used for the prediction of the dispersion characteristics at higher frequencies. Many researchers have used this kind of classical theories producing satisfying outcomes, such as Leppington et al. $[5,6]$ who modelled the radiation efficiency and the vibroacoustic response under a reverberant field. Others $[7,8]$ have mathematically improved the existing equations and examined the vibrational behaviour of laminated plates. Kurtze and Waters [9] were the first to examine the wave dispersion of thick sandwich structures by developing an asymptotic model. In their assumptions, though, the core was called incompressible, which kept them from modelling the deformation of the panel in the thickness sense. Dym and Lang [10], using the kinematic assumptions of [11] developed a structural model for an infinite sandwich panel deriving the five equations of motion corresponding to the symmetric and antisymmetric motion of the panel. Sokolinsky et al. [12] developed a consistent theory (Higher-Order Shear Deformation Theory, HSDT) taking into account the core's shear deformation and Wang et al. $[13,14]$ used it to construct a structural model of an infinitely long sandwich 
panel in which the vibroacoustic response within an Statistical Energy Analysis (SEA) context was calculated. Wave propagation has been major object of intense research with numerous numerical methods being developed the last decades. Finnveden in [15] examined hollow beam structures and presented a method of calculating the wave dispersion in them. In [16] the authors used Spectral Finite Element (SFE) to predict the wave propagation characteristics, overcoming the thresholds of CLPT. In [17] the phase constant surfaces of periodic composite and stiffened structures was examined taking advantage of the periodicity using Periodic Structure Theory (PST) and Finite Elements (FE). In this work, an expression for the computing of the radiation efficiency was presented and the STL was expressed through the radiation and mechanical impedances of the structures. In [18] and [19] the authors used a multi-layer analytical model based on Mindlin theory to calculate the dispersion characteristics of layered structures. In this work, though, the symmetric mode of motion was not naturally expressed. The same authors [20] came back presenting an approach for taking into account the symmetric wave motion for thick panels. Wave Finite Element Method (WFEM) was firstly introduced in [21]. Its main aspect is that it takes advantage of the periodicity of the structure and using existing classical literature's periodic theory [2] manages to examine a structure's wave propagation by modelling only a small periodic part of it using $\mathrm{FE}$ for its analysis. This way the calculation of the wavenumbers and eigenvectors is achieved with considerably lower cost of time than the previous ones. WFEM has been used in one dimension [22, 23] and in two dimensions analyses [24] producing quite satisfying results. Using FE for the structure's modelling has given researchers the ability to broaden the potentials of the method, calculating loss factor [25] with the help of existing theories [26]. In addition to that, Manconi et al. [27] calculated the effect of the pre-stress on the loss factor and wavenumbers of structures using two dimensional WFEM. Chronopoulos et al. in [28] used wave dispersion results of WFEM to calculate a dynamic stiffness matrix for a honeycomb orthotropic sandwich panel, the results of which were validated experimentally. Also, the same authors in [29] and [30] using WFEM 
and SEA computed the broadband vibroacoustic response of composite shells and thick layered panels. Another use of WFEM is the examination of the potential band gaps in periodic structures. Domadiya et al.[23] used WFEM to model two different periodic beams to examine the band gaps and had the results certified with actual experiments. Droz et al. [31] proposed a mathematically improved version of the WFEM and calculated the wave propagation and band gaps in a periodically stiffened plate.

In this paper the effect of pre-stress on wave propagation and acoustical behaviour of laminates was examined. Two-dimensional WFEM was used to calculate the Sound Transmission Loss (STL) of thick structures by accounting for their symmetric and antisymmetric wave motion. Both non-stressed and prestressed scenarios were examined. Equations from [30] were used to compute the reverberant field STL of the structures directly derived by their SEA properties. Finally, the loss factor of each structure was calculated.

The paper is organized as follows: in section 2 the WFEM is described, along with the calculation of the loss factor and the pre-stressed stiffness matrix $\mathbf{K}_{s}$. In Section 3 the calculation of the main SEA quantities is presented. In Section 4 the analysis scenarios are presented, along with the numerical results. Finally, in Section 5 concluding remarks are written and in Section 6 some thoughts on future work are presented.

\section{The two dimensional WFEM}

\subsection{Stress stiffening}

In this work two different scenarios of pre-stressed structure were examined, as described in the next section. In these cases, pre-stress stiffness matrix $\mathbf{K}_{s}$ was calculated. Considering that a static analysis has been solved, the updated stiffness matrix $\mathbf{K}$ was calculated [32]:

$$
\mathbf{K}=\mathbf{K}_{0}+\mathbf{K}_{s}
$$


where $\mathbf{K}_{0}$ the original element stiffness matrix and:

$$
\mathbf{K}_{s}=\iiint \mathbf{G}^{T} \tau \mathbf{G} \mathrm{d} x \mathrm{~d} y \mathrm{~d} z
$$

where $\mathbf{G}$ is a matrix of shape function derivatives and $\tau$ is a matrix of the current Cauchy (true) stresses $\sigma$ in the global Cartesian system.

The updated matrix $\mathbf{K}$ was then used in WFEM to get the wavenumbers and eigenvectors of the pre-stressed structure.

[Figure 1 about here.]

\subsection{Description of the WFEM}

In this paper a laminate of $L_{x}$ length and $L_{y}$ width was examined. An FE model of a small segment of the laminate was created. This segment's length was $\mathrm{d} x$, while its width was $\mathrm{d} y$ (Fig.1). The segment was meshed using commercially available FEA software. The vector of degrees of freedom (dofs) $\mathbf{q}$ of the segment is given in terms of dofs by [24]

$$
\mathbf{q}=\left[\begin{array}{llll}
\mathbf{q}_{1}^{T} & \mathbf{q}_{2}^{T} & \mathbf{q}_{3}^{T} & \mathbf{q}_{4}^{T}
\end{array}\right]^{T}
$$

where $T$ denotes the transpose and $\mathbf{q}_{n}$ is the vector of nodal dofs of all the elements nodes which lie on the $n$th corner of the element [24]. Following the same logic, the vector of nodal force is given by

$$
\mathbf{f}=\left[\begin{array}{llll}
\mathbf{f}_{1}^{T} & \mathbf{f}_{2}^{T} & \mathbf{f}_{3}^{T} & \mathbf{f}_{4}^{T}
\end{array}\right]^{T}
$$

Conventional FE methods is then used to get the $\mathbf{M}$ and $\mathbf{K}$ matrices of the segment. Assuming time-harmonic behaviour and neglecting damping we have

$$
\left[\mathbf{K}-\omega^{2} \mathbf{M}\right] \mathbf{q}=\mathbf{f}
$$

Using Floquet theorem for a rectangular segment and taking edge 1 as reference we get

$$
\mathbf{q}_{2}=\lambda_{x} \mathbf{q}_{1}, \quad \mathbf{q}_{3}=\lambda_{y} \mathbf{q}_{1}, \quad \mathbf{q}_{4}=\lambda_{x} \lambda_{y} \mathbf{q}_{1}
$$


where

$$
\lambda_{x}=e^{-i k_{x} \mathrm{~d} x}, \quad \lambda_{y}=e^{-i k_{y} \mathrm{~d} y}
$$

with $\lambda_{x}$ and $\lambda_{y}$ being the phase constants and $k_{x}$ and $k_{y}$ being the wavenumbers. Thus

$$
\mathbf{q}=\boldsymbol{\Lambda}_{R} \mathbf{q}_{1}, \quad \boldsymbol{\Lambda}_{R}=\left\{\begin{array}{c}
\mathbf{I} \\
\lambda_{x} \mathbf{I} \\
\lambda_{y} \mathbf{I} \\
\lambda_{x} \lambda_{y} \mathbf{I}
\end{array}\right\}
$$

with $\mathbf{I}$ being the identity matrix. Assuming no external excitation, equilibrium at node 1 should give zero sum of nodal forces.

$$
\boldsymbol{\Lambda}_{L}\left\{\begin{array}{c}
\mathbf{f}_{1} \\
\mathbf{f}_{2} \\
\mathbf{f}_{3} \\
\mathbf{f}_{4}
\end{array}\right\}=0, \quad \boldsymbol{\Lambda}_{L}=\left[\begin{array}{llll}
\mathbf{I} & \lambda_{x}^{-1} \mathbf{I} & \lambda_{y}^{-1} \mathbf{I} & \lambda_{x}^{-1} \lambda_{y}^{-1} \mathbf{I}
\end{array}\right]
$$

Dynamic stiffness $\mathbf{D}$ is introduced, which is

$$
\mathbf{D}=\mathbf{K}-\omega^{2} \mathbf{M}
$$

In case of damping, viscous or structural damping can be taken into account by the addition of viscous or structural damping matrices $\mathbf{C}$ or $\mathbf{K}_{I}$. Then, dynamic stiffness matrix is given by the following equations

$$
\mathbf{D}=\mathbf{K}+i \omega \mathbf{C}-\omega^{2} \mathbf{M}, \quad \text { and } \quad \mathbf{D}=\mathbf{K}+i \mathbf{K}_{I}-\omega^{2} \mathbf{M}
$$

Following the analysis in [24] and substituting Eq.(8) and Eq.(9) in Eq.(5) we get

$$
\mathbf{D}\left(\omega ; \lambda_{x}, \lambda_{y}\right) \mathbf{q}_{1}=0
$$


If the segment dynamic stiffness matrix is partitioned to

$$
\mathbf{D}=\left[\begin{array}{llll}
\mathbf{D}_{11} & \mathbf{D}_{12} & \mathbf{D}_{13} & \mathbf{D}_{14} \\
\mathbf{D}_{21} & \mathbf{D}_{22} & \mathbf{D}_{23} & \mathbf{D}_{24} \\
\mathbf{D}_{31} & \mathbf{D}_{32} & \mathbf{D}_{33} & \mathbf{D}_{34} \\
\mathbf{D}_{41} & \mathbf{D}_{42} & \mathbf{D}_{43} & \mathbf{D}_{44}
\end{array}\right]
$$

then the eigenproblem of Eq.(12) can be written in the following form

$$
\begin{array}{r}
{\left[\left(\mathbf{D}_{11}+\mathbf{D}_{22}+\mathbf{D}_{33}+\mathbf{D}_{44}\right)+\left(\mathbf{D}_{12}+\mathbf{D}_{34}\right) \lambda_{x}+\left(\mathbf{D}_{21}+\mathbf{D}_{43}\right) \lambda_{x}^{-1}\right.} \\
+\left(\mathbf{D}_{13}+\mathbf{D}_{24}\right) \lambda_{y}+\left(\mathbf{D}_{31}+\mathbf{D}_{42}\right) \lambda_{y}^{-1}+\mathbf{D}_{14} \lambda_{x} \lambda_{y}+\mathbf{D}_{41} \lambda_{x}^{-1} \lambda_{y}^{-1} \\
\left.+\mathbf{D}_{32} \lambda_{x} \lambda_{y}^{-1}+\mathbf{D}_{23} \lambda_{x}^{-1} \lambda_{y}\right] \mathbf{q}_{1}=0
\end{array}
$$

In [17] and [24] different methods were presented for solving the eigenproblem of Eq.(14). In this paper the method that was used in [30] was preferred. In [30] the frequency and the wavenumber towards $y$ direction were kept fixed and for each set of $\omega, k_{x}$ the $k_{y}$ values were calculated. In addition to this, by interpolating on the known results values for intermediate $\omega, k_{x}$ and $k_{y}$ can be found. For a set of fixed $\omega, k_{y}$ the non-linear eigenproblem of Eq.(14) can be reduced to

$$
\left(\mathbf{A}_{2} \lambda_{x}^{2}+\mathbf{A}_{1} \lambda_{x}+\mathbf{A}_{0}\right) \mathbf{u}_{Q}=0
$$

where

$$
\mathbf{A}_{i}=\left\{\begin{array}{l}
\mathbf{D}_{14} \lambda_{y}^{2}+\left(\mathbf{D}_{12}+\mathbf{D}_{34}\right) \lambda_{y}+\mathbf{D}_{32}, \quad i=2 \\
\left(\mathbf{D}_{13}+\mathbf{D}_{24}\right) \lambda_{y}^{2}+\left(\mathbf{D}_{11}+\mathbf{D}_{22}+\mathbf{D}_{33}+\mathbf{D}_{44}\right) \lambda_{y}+\mathbf{D}_{31}+\mathbf{D}_{42}, i=1 \\
\mathbf{D}_{23} \lambda_{y}^{2}+\left(\mathbf{D}_{21}+\mathbf{D}_{43}\right) \lambda_{y}+\mathbf{D}_{41}, \quad i=0
\end{array}\right.
$$

This quadratic eigenproblem can be converted [30] into and ordinary linear 
generalized eigenproblem, by introducing a new vector $\mathbf{z}=\lambda_{y} \mathbf{u}_{Q}$

$$
\left[\begin{array}{cc}
-\mathbf{A}_{0} & 0 \\
0 & \mathbf{I}
\end{array}\right]\left\{\begin{array}{c}
\mathbf{u}_{Q} \\
\mathbf{z}
\end{array}\right\}=\lambda_{y}\left[\begin{array}{cc}
\mathbf{A}_{1} & \mathbf{A}_{2} \\
\mathbf{I} & 0
\end{array}\right]\left\{\begin{array}{c}
\mathbf{u}_{Q} \\
\mathbf{z}
\end{array}\right\}
$$

Then we have

$$
k_{x}=\frac{\log \left(\lambda_{x}\right)}{-i \mathrm{~d} x} \quad \text { and } \quad k_{y}=\frac{\log \left(\lambda_{y}\right)}{-i \mathrm{~d} y}
$$

\subsection{Loss factor}

As it is written in Sec. 2 damping can be included in the dynamic stiffness matrix D in WFEM. In this work, though, our attention was focused on propagating waves in the undamped structure, whose wavenumbers were real (not complex) numbers and the loss factor as damping measurement.

A physical interpretation of the loss factor is expressed as followed [33]:

$$
\eta=\frac{1}{2 \pi} \frac{W_{d}}{U_{m}}=\frac{1}{2 \pi} \frac{\text { energy dissipated per cycle }}{\text { maximum strain energy }}
$$

Following the analysis in [25], the loss factor $\eta_{j}$ as a function of the frequency was calculated:

$$
\eta_{j}(\omega, T, \phi)=\frac{\mathbf{V}_{j}^{*} \mathbf{K}_{\omega, T, \phi}^{\prime \prime} \mathbf{V}_{j}}{\mathbf{V}_{j}^{*} \mathbf{K}_{\omega, T, \phi}^{\prime} \mathbf{V}_{j}}
$$

where $*$ stands for the conjugate transpose, $T$ for the temperature, $\phi$ for the angle that is examined, $\mathbf{V}_{j}$ is the displacement vector associated with the $j$ th propagating wave and is obtained from the wave mode $\mathbf{q}_{1 j}[24]$ and the components of the wavenumber $k_{x j}$ and $k_{y j}$ give:

$$
\mathbf{V}_{j}=\left[\begin{array}{llll}
\mathbf{q}_{1 j}^{T} & e^{-i k_{x j} L_{x}} \mathbf{q}_{1 j}^{T} & e^{-i k_{y j} L_{y}} \mathbf{q}_{1 j}^{T} & e^{-i k_{x j} L_{x}} e^{-i k_{y j} L_{y}} \mathbf{q}_{1 j}^{T}
\end{array}\right]^{T}
$$

In our case temperature was not accounted for. So we have

$$
\eta_{j}(\omega, \phi)=\frac{\mathbf{V}_{j}^{*} \mathbf{K}_{\omega}^{\prime \prime} \mathbf{V}_{j}}{\mathbf{V}_{j}^{*} \mathbf{K}_{\omega}^{\prime} \mathbf{V}_{j}}
$$


Also, it is used, along with $\mathbf{K}_{s}$ to calculate the loss factor $\eta_{s(j)}$ of the prestressed structure [27]:

$$
\eta_{s(j)}(\omega, \phi)=\frac{\mathbf{V}_{j}^{*} \mathbf{K}_{\omega}^{\prime \prime} \mathbf{V}_{j}}{\mathbf{V}_{j}^{*}\left(\mathbf{K}_{\omega}+\mathbf{K}_{s(\omega)}\right) \mathbf{V}_{j}}
$$

\section{Computation of the energy analysis quantities}

\subsection{Calculation of the modal density}

Using the Courant's formula [34], an equation was produced which expressed the modal density of each propagating wave type $w$ for each angle $\phi$ as a function of the propagating wavenumber and its corresponding group velocity $c_{g}$

$$
n_{w}(\omega, \phi)=\frac{A k_{w}(\omega, \phi)}{2 \pi^{2}\left|c_{g, w}(\omega, \phi)\right|}
$$

where $A$ was the area of the panel and the group velocity was expressed as

$$
c_{g}(\omega, \phi)=\frac{\mathrm{d} \omega}{\mathrm{d} k(\omega, \phi)}
$$

Then, the modal density after having been angularly averaged was given as a function of frequency:

$$
n_{w}(\omega)=\int_{0}^{\pi} n_{w}(\omega, \phi) \mathrm{d} \phi
$$

\subsection{Calculation of the radiation efficiency}

Radiation efficiency $\sigma(k(\omega))$ for each propagating wave type $w$ was calculated using existing equations in open literature. Mode shapes of sinusoidal form can be assumed for continuous structures so that any FE discretization errors to be avoided. For the calculation of $\sigma(k(\omega))$ the set of asymptotic formulas given in [5] was used. Working in an SEA context, energy equipartition amongst the resonant modes was assumed so that:

$$
\sigma_{\operatorname{rad}(\omega)}=\frac{1}{n(\omega)} \int_{0}^{\pi} \sigma(\kappa(\omega, \phi)) n(\omega, \phi) \mathrm{d} \phi
$$


For a periodic discontinuous structure the assumption of sinusoidal mode shapes is no longer valid, which means that the radiation efficiency should be calculated from the WFEM derived wave mode shapes. Therefore, the radiation efficiency expression given in [17] can be used. In the next chapter the equations used to calculate the STL, modal density and radiation efficiency are presented.

3.3. Calculation of the Sound Transmission Loss (STL) of a panel by a SEA approach

The STL (or TL) is considered to be a property of great importance which is crucial to examine and quantify the characterisation of the vibroacoustic performance of a structure. The system examined in this work consisted of two reverberant chambers separated by the modelled panel which was attached to a rigid baffle. Any flanking transmission was neglected in the SEA model. In Fig.2 a graphical representation of the model is depicted, along with the energy balance of the subsystems of the SEA approach [35], where $E_{1}$ and $E_{3}$ are the acoustic energy of the source room and the receiving room respectively and $E_{2}$ is the vibrational energy of the panel. $P_{i n}$ is the injected power in the source room, $P_{1 d}, P_{2 d}$ and $P_{3 d}$ are the power dissipated by each sub-system and $P_{13}$ is the non-resonant transmitted power between the rooms.

[Figure 2 about here.]

Considering each wave type $w=a, b, c \ldots n$ propagating within the panel as a separate SEA subsystem:

$$
\begin{aligned}
& P_{12}=\sum_{w=a}^{n} P_{12, w} \\
& P_{23}=\sum_{w=a}^{n} P_{23, w}
\end{aligned}
$$

where $P_{12}$ and $P_{23}$ stand for the power flow between the rooms and the panel. 
The STL was calculated by the equation:

$$
S T L=10 \log _{10}\left(\frac{1}{\tau}\right)
$$

where $\tau$ is the transmission coefficient which represents the ratio between the transmitted and the incident sound powers. $\tau$ can be written as the sum of the resonant and the non-resonant transmission coefficient

$$
\tau=\frac{P_{23}+P_{13}}{P_{i n c}}=\sum_{w=a}^{n} \frac{P_{23, w}}{P_{i n c}}+\frac{P_{13}}{P_{i n c}}
$$

where $P_{i n c}$ is the acoustic power incident on the panel, which for a reverberant sound field can be expressed as:

$$
P_{\text {inc }}=\frac{\left\langle p_{1}^{2}\right\rangle A}{4 p c}
$$

where $\left\langle p_{1}^{2}\right\rangle$ is the mean-square sound pressure. Using the calculations made in [30] the resonant coefficient for each wave type $w$ was examined. Assuming no energy exchanges between different wave types within the panel, the energy balance of a structural wave subsystem can be expressed as:

$$
P_{12, w}=P_{2 d, w}+P_{23, w}
$$

The power dissipated was:

$$
P_{2 d, w}=E_{2, w} \omega \eta_{2, w}
$$

with $\eta_{2, w}$ being the structural loss factor of the wave type $w$. The loss factor is a known property of most of isotropic materials and can be calculated in layered structures if each layers' loss factor is known [36]. In this paper, as shown in Sec. 2.3 the loss factor was calculated as a function of frequency for both non-stressed and pre-stressed analyses. The vibrational energy of the panel due to wave type $w$ was written as:

$$
E_{2, w}=\rho_{s} A\left\langle v_{w}^{2}\right\rangle
$$


where $\rho_{s}$ was the mass per unit of area, $A$ was the total area of the panel and $\left\langle v_{w}^{2}\right\rangle$ was the mean-square panel vibration velocity due to wave type $w$. The power flow $P_{12, w}$ can be written using the SEA reciprocity rule as:

$$
P_{12, w}=\omega \eta_{12, \omega} n_{1}\left(\frac{E_{1}}{n_{1}}-\frac{E_{2, w}}{n_{2, w}}\right)=\omega \eta_{21, w} n_{2, w}\left(\frac{E_{1}}{n_{1}}-\frac{E_{2, w}}{n_{2, w}}\right)
$$

where $n_{1}$ and $n_{2, w}$ are the modal density of the source room and of the wave type $w$ respectively and $\eta_{21, w}$ the coupling loss factor between the receiving room and the wave type $w$ which was written as:

$$
\eta_{21, w}=\eta_{23, w}=\frac{\rho c \sigma_{r a d, w}}{\rho_{s} \omega}
$$

where $\rho$ is the acoustic medium density of the room. The total acoustic energy of the source room is:

$$
E_{1}=\frac{\left\langle p_{1}^{2}\right\rangle V}{\rho c^{2}}
$$

Following the analysis in [30], the total transmission coefficient of the panel $t$ was expressed as:

$$
\tau=\sum_{\omega=a}^{n} \tau_{\omega}+\frac{P_{13}}{P_{i n c}}
$$

where

$$
\tau_{w}=\frac{8 \rho^{2} c^{4} \pi \sigma_{r a d, w}^{2} n_{2, w}}{\rho_{s} \omega^{2} A\left(\rho_{s} \omega n_{2, w}+2 \rho c \sigma_{r a d, w}\right.}
$$

The non resonant transmission coefficient $\tau_{n r}=P_{13} / P_{i n c}$ for a diffused acoustic field was written as:

$$
\frac{P_{13}}{P_{\text {inc }}}=\frac{\int_{0}^{2 p i} \int_{0}^{\theta_{\operatorname{mac}}} \frac{4 Z_{0}^{2} \sigma(\theta, \phi, \omega) \sin \theta \cos ^{2} \theta}{\left|i \omega \rho_{s}+2 Z_{0}\right|^{2}} \mathrm{~d} \theta \mathrm{d} \phi}{\pi\left(1-\cos ^{2} \theta_{\max }\right)}
$$

in which $\theta$ and $\phi$ were the incidence angle and the direction angle of the acoustic wave respectively, and $Z_{0}=\rho c / \cos \theta$ was the acoustic impedance of the medium. The term $\theta_{\max }$ was the maximum incidence angle, accounting for 
the diffuseness of the incident field. In this paper $\theta_{\max }=p i / 2$ was considered. The term $\sigma(\theta, \phi, \omega)$ is the corrected radiation efficiency term, which was used so that to account for the finite dimensions of the panel by accounting for the radiation of the mass controlled non-resonant modes, and it was calculated using a spatial windowing correction technique presented in [37]. In Eq.(38) the total transmission coefficient of the panel is expressed merely as a function of its SEA quantities and independently of the room dimensions and modal energies. In the next section the numerical results of the different scenarios and laminates examined are presented.

\section{Numerical results}

Two different laminates, a monolithic and a sandwich, were examined. Both of them were simulated under a scenario in which the plates were tensioned and compressed. The sandwich laminate was additionally examined in a different case where internal pressure was applied in its core, as it is shown in Fig.15. The mechanical testing characteristics of each material used in the models are listed

in Table 1 , where $E_{i}$ is the modulus of elasticity in direction $i, v_{i j}$ is the Poisson's ratio for $i$ and $j$ being the directions of extension and contraction, respectively, $\rho$ is the density and $G_{i j}$ is the shear modulus of elasticity in direction $j$ on the plane whose normal is in direction $i$. ANSYS 14.0 was used during the FE modelling. Linear 8-node ANSYS SOLID45 solid element was chosen for the segment's meshing, which comprises a 3D displacement field and three degrees of freedom per node (translations in the $\mathrm{x}, \mathrm{y}$, and $\mathrm{z}$ directions) [32]. The maximum element size was kept below one tenth of the length of the minimum structural wave propagating in the frequency range of the analysis so that any interpolation errors were avoided.

[Table 1 about here.]

\subsection{Monolithic laminate - Tensioned and Compressed}

[Figure 3 about here.] 
Three different scenarios were examined: one with no force applied on the laminate, one with $\mathrm{T}=100 \mathrm{~N}$ tensile force applied in $x$ direction and one with $\mathrm{T}=100 \mathrm{~N}$ compressive force in $x$ direction. Its dimensions were $L x=2.5 \mathrm{~m}$, $L y=1.5 m$ the length and width, and $h=0.002 m$ its thickness (Fig.3). Material $I$ (Table 1) was used in the analysis. The flexural wavenumbers in $x$ direction of all three scenarios are presented in Fig.4. It can be seen that tension decreases the magnitude of the wavenumber, while compression increases it. The same behaviour is noticed in the loss factor behaviour in Fig.5. This can be explained by the fact that in the first case the structure is stiffened, with the strain energy associated with the tension increased (in comparison with the bending one) resulting in decreasing of the loss factor (see Eq.(19)). Eq.(23), also, explains this behaviour since $\mathbf{K}_{s}$ consists of real positive numbers when the laminate is tensioned (thus stiffened) and real negative numbers when the laminate is compressed (thus softened). In Manconi's work [27] on loss-factor of pre-stressed structures, results of the same nature were acquired examining pre-stressed beams and cylinders. It should be noted that these differences are normalized as frequency gets higher, with $1 \mathrm{kHz}$ being the frequency where the values tend to become equal. The same behaviour is noticed in most of the rest graphs.

[Figure 4 about here.]

[Figure 5 about here.]

In Fig.6 the modal density of the three scenarios are depicted. It can be seen that, although both pre-stressed cases exhibit different behaviour than the nonstressed one, their results are identical in tensioned and compressed laminate.

[Figure 6 about here.]

On the other hand, in Fig.7 we see that in tensioned case the radiation efficiency is higher than in non-stressed case, while in compressed case the opposite is obvious, with radiation efficiency having lower values. As mentioned before, these differences are normalized as frequency gets higher. 
[Figure 7 about here.]

In Fig.8 the TL of the three scenarios are shown. Again, in tensioning case the TL is lower that the non-stressed in low frequencies. In compressive case, though, there is no significant difference compared to the non-stressed.

[Figure 8 about here.]

\subsection{Sandwich laminate}

[Figure 9 about here.]

A sandwich laminate with the same dimensions as the monolithic one (length $L_{x}=2.5 m$, width $L_{y}=1.5 m$ ) was modelled. Material $I I$ was used for the skin and Material III for the core. The core's thickness was $h_{c}=0.0127 \mathrm{~m}$ and the skins' one was $h_{s}=0.0012 m$ (Fig.9).

\subsubsection{Tensioned-Compressed}

As in Subsec. 4.1 three different scenarios were examined, with no force applied on the sandwich laminate and both tension and compression, $T= \pm 100 N$. In Fig.10 the wavenumbers are depicted. In this case the same behaviour as in Fig.4 is noticed, but the differences are of smaller scale.

[Figure 10 about here.]

In Fig.11 the loss factors are depicted. Again it is shown that the same behaviour as in Fig.5 is noticed in sandwich laminate occasion. As it was written in 4.1 , this is explained by the change in strain energy of the laminate.

[Figure 11 about here.]

In Fig.12, 13 and 14 the modal density, radiation efficiency and TL distributions are shown. In this cases the differences between the three scenarios are quite insignificant. Also, it should be noted that all three of them are characterised by smoother curves than the monolithic laminate ones. 
[Figure 12 about here.]

[Figure 13 about here.]

[Figure 14 about here.]

\subsubsection{Internally pressurised}

[Figure 15 about here.]

The second case of the sandwich laminate consists of internal pressure in the core, as it is shown in Fig.15. Two different pressures, $p=10 \mathrm{bar}$ and $p=100 \mathrm{bar}$ were applied. In all the curves it can be seen that the first pressure scenario's results were quite close to the non-pressurised one's, as opposed to the second pressure scenario's ones. More specifically, in Fig.16 the wavenumbers of the three scenarios are depicted. In this case, in low frequencies the wavenumbers of the high pressurised sandwich are significantly different than the low pressurised and the non-pressurised. The same behaviour is noticed in Fig.17. Having Eq.(19) in mind, this behaviour can be explained, since when internal pressure is applied to the sandwich laminate's core, its strain energy is increased, thus the loss factor is decreased. Also, the pressure in the second pressure scenario is higher, and so is the strain increase which leads to lower loss factor. Another behaviour that it is noticed is that in the higher pressure scenario the loss factor remains lower than the non-pressurised all over the frequency range, with the difference diminishing as the frequency gets higher, though.

[Figure 16 about here.]

[Figure 17 about here.]

In Fig.18, 19 and 20, the same behaviour is noticed in modal density, radiation efficiency and TL dispersion curves. In these results it should, also, be noted that the higher pressure depicts different numerical results from the nonpressurised and the low pressure scenario through almost the whole frequency range that was examined. 
[Figure 18 about here.]

[Figure 19 about here.]

[Figure 20 about here.]

Finally, in Fig.21 and 22 the tensioned and pressurised results of the sandwich plate are compared. It can be seen that 10bar pressure and $100 \mathrm{~N}$ tensile force had almost identical effect on the loss factor and TL of the plate. More particularly, both scenarios' maximum TL reduction was $\sim 2.8 d B(15 \%)$ and at the same frequency $(\sim 950 \mathrm{~Hz})$, while pressurised sandwich's loss factor reduction was $\sim 0.03(33 \%)$ and the tensioned one's was $\sim 0.02(21 \%)$ at low frequencies. On the other hand, 100bar pressure affects largely both magnitudes. Concerning TL, the maximum decrease was $\sim 10 d B(43 \%)$ and it should be noted that it is observed at different frequency $(\sim 650 \mathrm{~Hz})$ than the other two scenarios. As for the loss factor, the decrease was quite large, with $\sim 0.07(88 \%)$ being the largest one at low frequencies. This behaviour of the pressurised and tensioned sandwich laminates raises some concerns about the vibroacoustic performance of pre-stressed structures which has not been widely examined.

[Figure 21 about here.]

[Figure 22 about here.]

\section{Conclusions}

The effect of pre-stress on the wave propagation and acoustic transmission properties of pressurised and pre-stressed composite structures was examined in this paper. WFEM was used for the modelling of two different laminates, a monolithic and a sandwich one. The pre-stress effect was calculated using commercially available FE software, extracting the pre-stressed stiffness matrices and using them in WFEM. The monolithic laminate was examined in both tension and compression, while the sandwich laminate was examined in internal (core) pressure scenarios, too, along with the tension and compression 
ones. The loss factor and important SEA quantities were calculated, namely the modal density, the TL and the radiation efficiency of the panels following the calculation of their dispersion characteristics. Existing literature equations were used for these calculations.

It was noticed that pre-stress affects the loss factor and the wave dispersion curves, which results in the alteration of the modal density, radiation efficiency and TL behaviour. More specifically, it was shown that the tension and compression of the laminates alter the wave dispersion and loss factor in low frequencies, with the monolithic laminate case depicting more significant alterations in the results. In the internal core pressure of the sandwich, it was shown that if the pressure is high enough, quite significant alteration in vibroacoustic behaviour can be achieved, potentially in wide range of frequency.

\section{Future work}

The WFEM taking into account the pre-stress effect on the structures offers wide range of potentials concerning the vibroacoustic analysis of structures. The effect of different scenarios of pre-stress can be examined, such as hydrothermal stress. Also, as Manconi et al. [27] did concerning cylindrical pressure vessels, research might focus on the adaptation of the method on more complex geometries. Another promising aspect of WFEM is the research of the possible effect of pre-stress on band-gaps, since the method has already been used in [23] and [31] on stiffened plates and beams. Wave dispersion graph of periodically pressured beam has been generated (see Fig.23), with the stress exceeding known materials' limits, though, without any useful conclusions yet. Nevertheless, these results are the base of current research on band-gaps on periodically pressurised structures.

[Figure 23 about here.] 


\section{References}

[1] K. F. Graff, Wave motion in elastic solids, 1975.

[2] L. Brillouin, Wave propagation in periodic structures, 1946.

[3] J. N. Reddy, Mechanics of laminated composite plates: theory and analysis, 1997.

[4] J. M. Whitney, N. J. Pagano, Shear deformation in heterogeneous anisotropic plates, Journal of Applied Mechanics 37 (1970) 1031-1036. doi:10.1115/1.3408654.

[5] F. G. Leppington, E. G. Broadbent, K. H. Heron, The acoustic radiation efficiency of rectangular panels, Proceedings of the Royal Society of London 382 (1982) 245-271. doi:10.1098/rspa.1982.0100.

[6] F. G. Leppington, K. H. Heron, E. G. Broadbent, Resonant and nonresonant ransmission of random noise through complex plates, Proceedings of the Royal Society of London 458 (2002) 245-271. doi:10.1098/rspa. 2001.0870 .

[7] S. S. Alielding, A. E. Alshorbagy, M. Shaat, A first-order shear deformation finite element model for elastostatic analysis of laminated composite plates and the equivalent functionally graded plates, Ain Shams Engineering Journal 2 (2011) 53-62. doi:10.1016/j.asej .2011.05.003.

[8] H. T. Thai, D. H. Choi, A simple first-order shear deformation theory for laminated composite plates, Composite Structures 106 (2013) 754-763. doi:10.1016/j. compstruct. 2013.06.013.

[9] G. Kurtze, B. G. Watters, New wall design for high transmission loss or high damping, The Journal of the Acoustical Society of America 31 (1959) 739-748. doi:10.1121/1.1907780. 
[10] C. L. Dym, M. A. Lang, Transmission of sound through sandwich panels, The Journal of the Acoustical Society of Amrica 56 (1974) 1523-1532. doi:10.1121/1.1903474.

[11] R. D. Ford, P. Lord, A. W. Walker, Sound transmission through sandwich constructions, Journal of Sound and Vibration 5 (1967) 9-21. doi:10. 1016/0022-460X (67) 90173-3.

[12] R. D. Ford, P. Lord, A. W. Walker, Consistent higher-order dynamic eqations for soft-core sandwich beams, AIAA Journal 42 (2004) 374-382.

[13] T. Wang, V. S. Sokolinsky, S. Rajaram, S. R. Nutt, Assessment of sandwich models for the prediction of sound transmission loss in unidirectional sandwich panels, Applied Acoustics 66 (2005) 245-262. doi:10.1016/j . apacoust.2004.08.005.

[14] T. Wang, S. Li, S. Rajaram, S. R. Nutt, Predicting the sound transmission loss of sandwich panels by statistical energy analysis approach, Journal of Vibration and Acoustics 132 (2010) 011004. doi:10.1115/1.4000459.

[15] S. Finnveden, Evaluation of modal density and group velocity by a finite element method, Journal of Sound and Vibration 273 (2004) 51-75. doi: $10.1016 / \mathrm{j} \cdot \mathrm{jsv} \cdot 2003.04 .004$.

[16] E. Barbieri, A. Cammarano, S. D. Rosa, F. Franco, Waveguides fo a composite plate by using the Spectral Finite Element Approach, Journal of Vibration and Control 15 (2009) 347-367.

[17] V. Cotoni, R. S. Langley, P. J. Shorter, A statistical energy analysis subsystem formulation using finite element and periodic structure theory, Journal of Sound and Vibration 318 (2008) 1077-1108. doi:10.1016/j.j sv. 2008. 04.058.

[18] S. Ghinet, N. Atalla, Vibro-acoustic behaviors of flact sandwich composite panels, Transactions of the Canadian Society for Mechanical Engineering 30 (2006) 473-493. 
[19] S. Ghinet, N. Atalla, . Osman, The transmission loss of curved laminates and sandwich composite panels, The Journal of the Acoustical Society of America 118 (2005) 774-790. doi:10.1121/1.1932212.

[20] S. Ghinet, N. Atalla, Modeling thick composite laminate and sandwich structures with linear viscoelastic damping, Computers and Structures 89 (2011) 1547-1561. doi:10.1016/j.compstruc.2010.09.008.

[21] D. J. Mead, A general theory of harmonic wave propagation in linear periodic systems with multiple coupling, Journal of Sound and Vibration 27 (1973) 235-260. doi:10.1016/0022-460X (73) 90064-3.

[22] B. R. Mace, D. Duhamel, M. J. Brennan, L. Hinke, Finite element prediction of wave motion in structural waveguides, Acoustical Society of America 117 (2005) 2835-2843. doi:10.1121/1.1887126.

[23] P. G. Domadiya, E. Manconi, M. Vanali, L. V. Andersen, A. Ricci, Numerical and experimental investigation of stop-bands in finite and infinite periodic one-dimensional structures, Journal of Vibration and Control (2014) 1-12doi : 10.1177/1077546314537863.

[24] B. R. Mace, E. Manconi, Modelling wave propagation in two-dimensional structures using finite element analysis, Journal of Sound and Vibration 318 (2008) 884-902. doi:10.1016/j.jsv.2008.04.039.

[25] E. Manconi, B. R. Mace, Estimation of the loss factor of viscoelastic laminated panels from finite element analysis, Journal of Sound and Vibration 329 (2010) 3928-3939. doi:10.1016/j.jsv.2010.04.014.

[26] C. Johnson, D. A. Kienholz, Finite element prediction of damping in structures with constrained viscoelastic layers, AIAA Journal 20 (1982) 12841290.

[27] E. Manconi, B. R. Mace, R. Garziera, The loss-factor of pre-stressed laminated curved panels and cylinders using a wave and finite element 
method, Journal of Sound and Vibration 332 (2013) 1704-1711. doi: $10.1016 / j \cdot j s v \cdot 2012.09 .039$.

[28] D. Chronopoulos, M. Ichchou, B. Troclet, On the vibroacoustic response of aerospace sandwich structures: Bridging the gap between experimental and numerical results, Proceedings of ISMA2012, 2012.

[29] D. Chronopoulos, B. Troclet, M. Ichchou, J. P. Laine, A unified approach for the broadband vibroacoustic response of composite shells, Composites Part B: Engineering 43 (2012) 1837-1846. doi:10.1016/j.compositesb. 2012.01 .059 .

[30] D. Chronopoulos, M. Ichchou, B. Troclet, O. Bareille, Computing the broadband vibroacoustic response of arbitrarily thick layered panels by a wave finite element approach, Applied Acoustics 77 (2014) 89-98. doi: $10.1016 / j$.apacoust. 2013.10.002.

[31] C. Droz, C. Zhou, M. N. Ichchou, J. P. Laine, A hybrid wave-mode formulation for the vibro-acoustic analysis of 2D periodic structures, Journal of Sound and Vibration 363 (2016) 285-302. doi:10.1016/j.jsv.2015.11. 003.

[32] ANSYS, Inc., ANSYS 14.0 Help file (2011).

[33] M. Petyt, Introduction to Finite Element Vibration Analysis, 1990.

[34] R. Courant, D. Hilbert, Methods of mathematical physics, vol. 1, 1989.

[35] R. H. Lyon, R. G. DeJong, M. Heckl, Theory and application of Statistical Energy Analysis, 1995. doi:10.1121/1.413875.

[36] P. J. Shorter, Wave propagation and damping in linear viscoelastic laminates, Journal of the Acoustical Society of America 115 (2004) 1917-1925. doi:10.1121/1.1689342.

[37] J. Allard, N. Atalla, Propagation of Sound in Porous Media: Modelling Sound Absorbing Materials 2e, 2009. 


\section{List of Figures}

1 Representation of the modelled internally pressurised periodic segment with its edges $1,2,3$ and 4 . . . . . . . . . . . 25

2 The SEA model . . . . . . . . . . . . . . . . . 26

3 Laminate ... . . . . . . . . . . . . . . . 27

4 Comparison of the bending wavenumber (x-direction) of the monolithic laminate: (-) non-stressed, (x) pre-stressed, tensile force $\mathrm{T}=100 \mathrm{~N}$ in $x$ direction, (o) pre-stressed, compressive force $\mathrm{T}=-$ $100 \mathrm{~N}$ in $x$ direction . . . . . . . . . . . . . . . . . . 28

5 Comparison of the loss factor of the monolithic laminate: (-) nonstressed, (x) pre-stressed, tensile force $\mathrm{T}=100 \mathrm{~N}$ in $x$ direction, (o) pre-stressed, compressive force $\mathrm{T}=-100 \mathrm{~N}$ in $x$ direction . . . . . . 29

6 Comparison of the modal density of the monolithic laminate: (-) non-stressed, (x) pre-stressed, tensile force $\mathrm{T}=100 \mathrm{~N}$ in $x$ direction, (o) pre-stressed, compressive force $\mathrm{T}=-100 \mathrm{~N}$ in $x$ direction . 30

7 Comparison of the radiation efficiency of the monolithic laminate: (-) non-stressed, (x) pre-stressed, tensile force $\mathrm{T}=100 \mathrm{~N}$ in $x$ direction, (o) pre-stressed, compressive force $\mathrm{T}=-100 \mathrm{~N}$ in $x$ direction 31

8 Comparison of the transmission loss of the monolithic laminate: (-) non-stressed, (x) pre-stressed, tensile force $\mathrm{T}=100 \mathrm{~N}$ in $x$ direction, (o) pre-stressed, compressive force $\mathrm{T}=-100 \mathrm{~N}$ in $x$ direction 32

9 Representation of the sandwich laminate . . . . . . . . . . . . 33

10 Comparison of the bending wavenumber (x-direction) of the sandwich plate: (-) non-stressed, (x) pre-stressed, tensile force $\mathrm{T}=100 \mathrm{~N}$ in $x$ direction, (o) pre-stressed, compressive force $\mathrm{T}=-100 \mathrm{~N}$ in $x$ direction ................... . . 34

11 Comparison of the loss factor of the sandwich plate: (-) nonstressed, (x) pre-stressed, tensile force $\mathrm{T}=100 \mathrm{~N}$ in $x$ direction, (o) pre-stressed, compressive force $\mathrm{T}=-100 \mathrm{~N}$ in $x$ direction . . . . 35

12 Comparison of the modal density of the sandwich plate: (-) nonstressed, (x) pre-stressed, tensile force $\mathrm{T}=100 \mathrm{~N}$ in $x$ direction, (o) pre-stressed, compressive force $\mathrm{T}=-100 \mathrm{~N}$ in $x$ direction . . . . 36

13 Comparison of the radiation efficiency of the sandwich plate: (-) non-stressed, (x) pre-stressed, tensile force $\mathrm{T}=100 \mathrm{~N}$ in $x$ direction, (o) pre-stressed, compressive force $\mathrm{T}=-100 \mathrm{~N}$ in $x$ direction .

14 Comparison of the transmission loss of the sandwich plate: (-) non-stressed, (x) pre-stressed, tensile force $\mathrm{T}=100 \mathrm{~N}$ in $x$ direction, (o) pre-stressed, compressive force $\mathrm{T}=-100 \mathrm{~N}$ in $x$ direction . 38

15 Representation of the internally pressurised sandwich laminate . 39

16 Comparison of the bending wavenumber (x-direction) of the sandwich plate: (-) non-pressurised, (x) pre-stressed, internal pressure $\mathrm{p}=10$ bar, (o) pre-stressed, internal pressure $\mathrm{p}=100 \mathrm{bar}$. . . . . .

17 Comparison of the loss factor of the sandwich plate: (-) nonpressurised, (x) pre-stressed, internal pressure $\mathrm{p}=10 \mathrm{bar},(\mathrm{o})$ prestressed, internal pressure $\mathrm{p}=100 \mathrm{bar}$. . . . . . . . . . . . 
18 Comparison of the modal density of the sandwich plate: (-) nonpressurised, (x) pre-stressed, internal pressure $\mathrm{p}=10 \mathrm{bar}$, (o) prestressed, internal pressure $\mathrm{p}=100 \mathrm{bar} \ldots \ldots \ldots . \ldots 42$

19 Comparison of the radiation efficiency of the sandwich plate: (-) non-pressurised, (x) pre-stressed, internal pressure $\mathrm{p}=10 \mathrm{bar}$, (o) pre-stressed, internal pressure $\mathrm{p}=100 \mathrm{bar} \ldots \ldots . . . \ldots 43$

20 Comparison of the transmission loss of the sandwich plate: (-) non-pressurised, (x) pre-stressed, internal pressure $\mathrm{p}=10 \mathrm{bar}$, (o) pre-stressed, internal pressure $\mathrm{p}=100 \mathrm{bar} \ldots \ldots \ldots . . \ldots 44$

21 Comparison of the transmission loss of the sandwich plate: () non-stressed, (x) pre-stressed, internal pressure $\mathrm{p}=10 \mathrm{bar}$, (o) pre-stressed, internal pressure $\mathrm{p}=100 \mathrm{bar},(+)$ pre-stressed, tensile force $\mathrm{T}=100 \mathrm{~N} \ldots \ldots \ldots \ldots \ldots \ldots \ldots$

22 Comparison of the transmission loss of the sandwich plate: () non-stressed, (x) pre-stressed, internal pressure $\mathrm{p}=10 \mathrm{bar}$, (o) pre-stressed, internal pressure $\mathrm{p}=100 \mathrm{bar},(+)$ pre-stressed, tensile force $\mathrm{T}=100 \mathrm{~N} \ldots \ldots \ldots \ldots \ldots \ldots \ldots$

23 Wave dispersion graph of periodically pressured beam $\ldots \ldots 47$ 


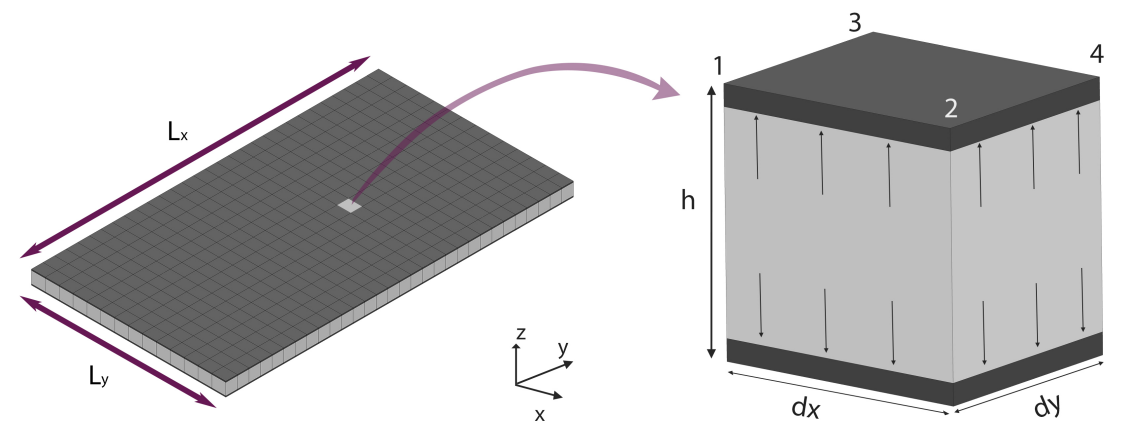

Figure 1: Representation of the modelled internally pressurised periodic segment with its edges $1,2,3$ and 4 

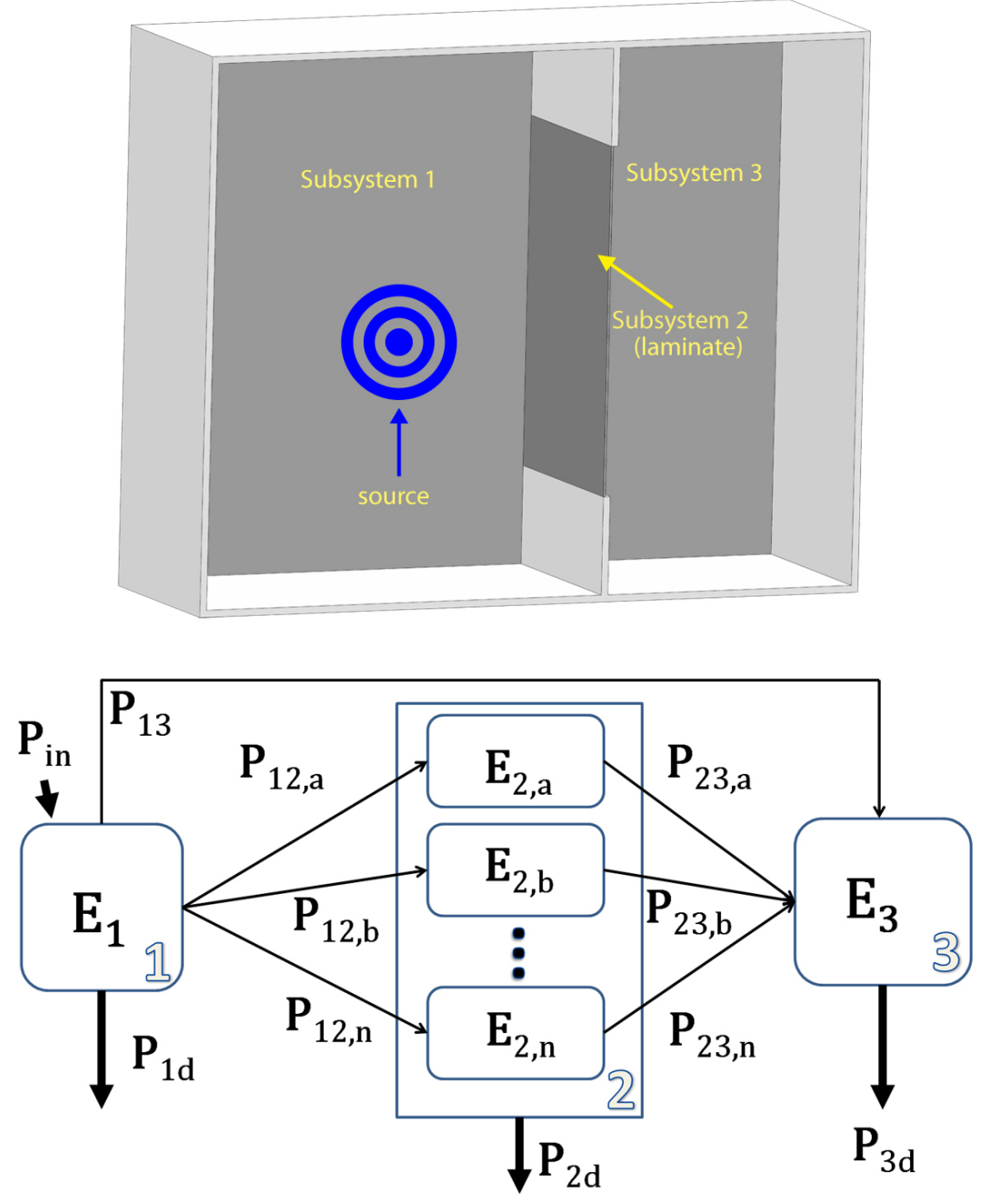

Figure 2: The SEA model 


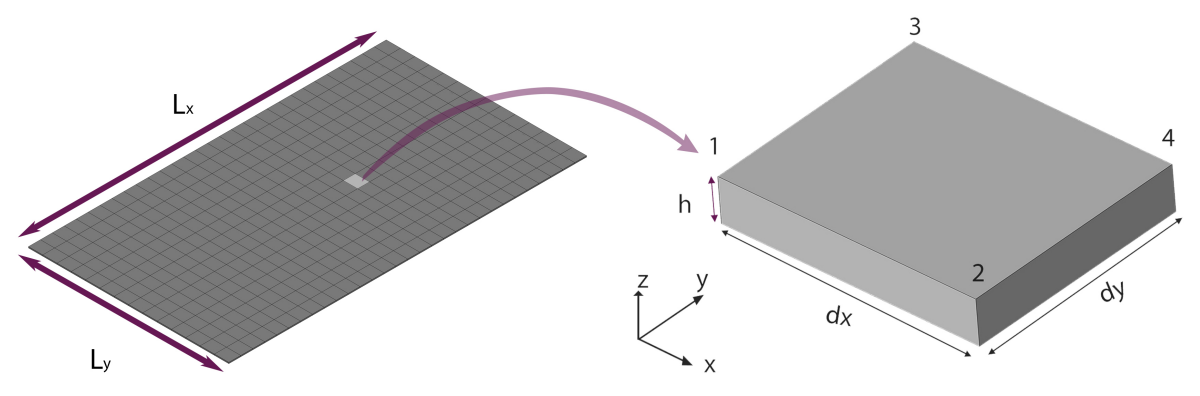

Figure 3: Laminate 


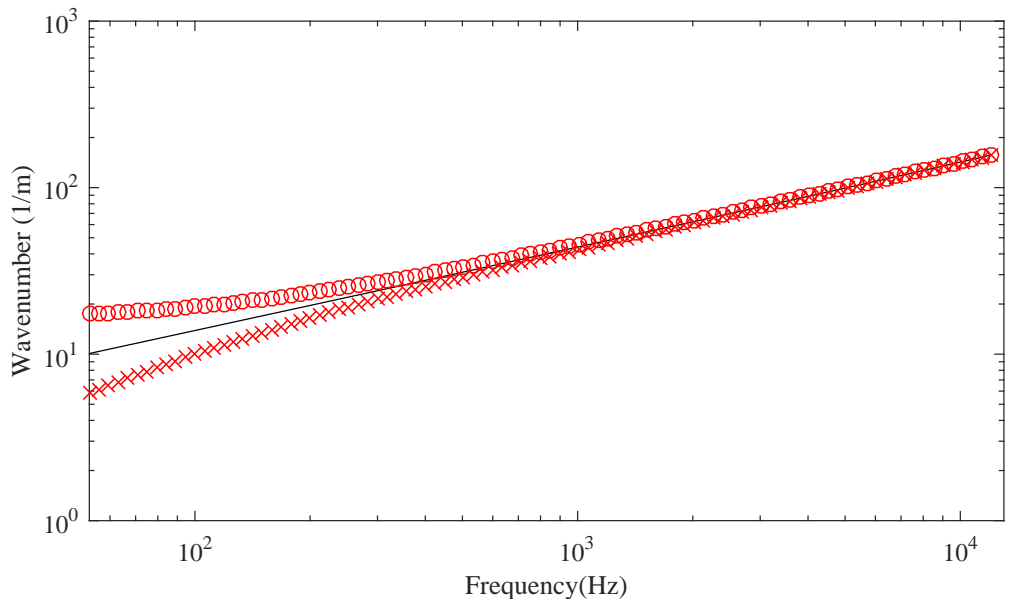

Figure 4: Comparison of the bending wavenumber (x-direction) of the monolithic laminate: (-) non-stressed, (x) pre-stressed, tensile force $\mathrm{T}=100 \mathrm{~N}$ in $x$ direction, (o) pre-stressed, compressive force $\mathrm{T}=-100 \mathrm{~N}$ in $x$ direction 


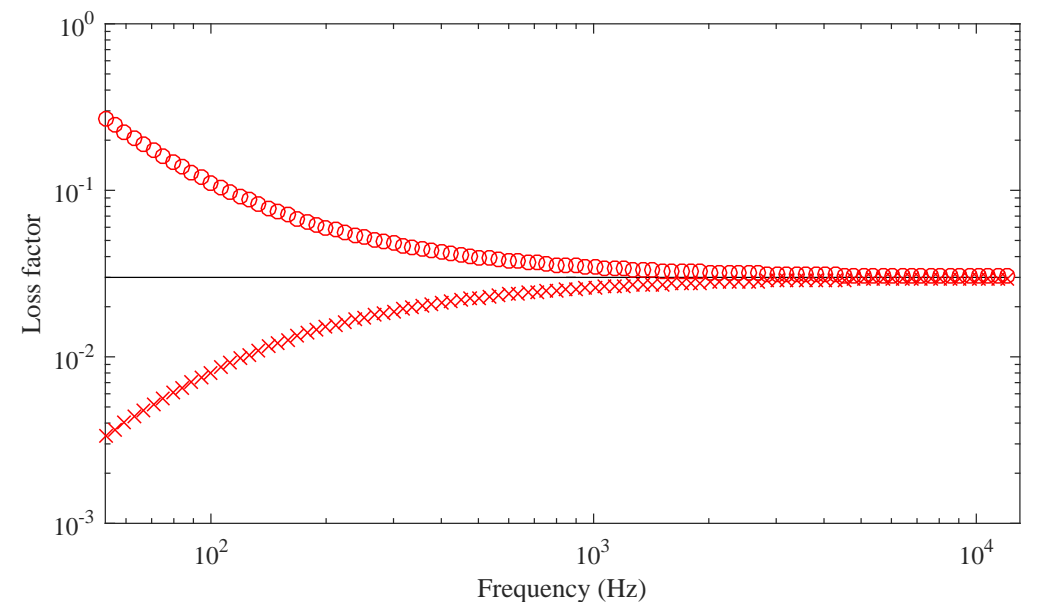

Figure 5: Comparison of the loss factor of the monolithic laminate: (-) non-stressed, (x) prestressed, tensile force $\mathrm{T}=100 \mathrm{~N}$ in $x$ direction, (o) pre-stressed, compressive force $\mathrm{T}=-100 \mathrm{~N}$ in $x$ direction 


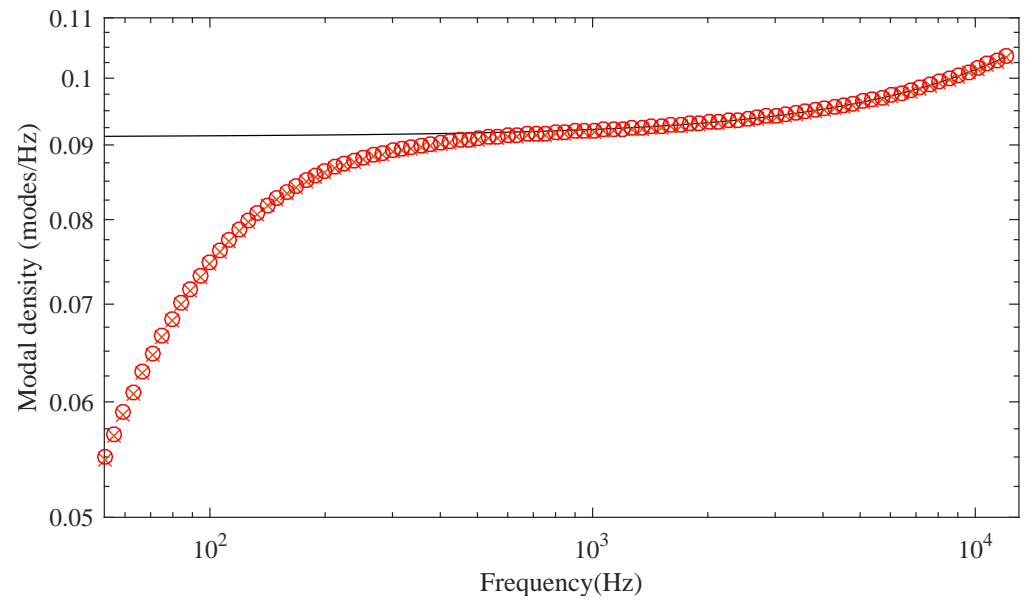

Figure 6: Comparison of the modal density of the monolithic laminate: (-) non-stressed, (x) pre-stressed, tensile force $\mathrm{T}=100 \mathrm{~N}$ in $x$ direction, (o) pre-stressed, compressive force $\mathrm{T}=-100 \mathrm{~N}$ in $x$ direction 


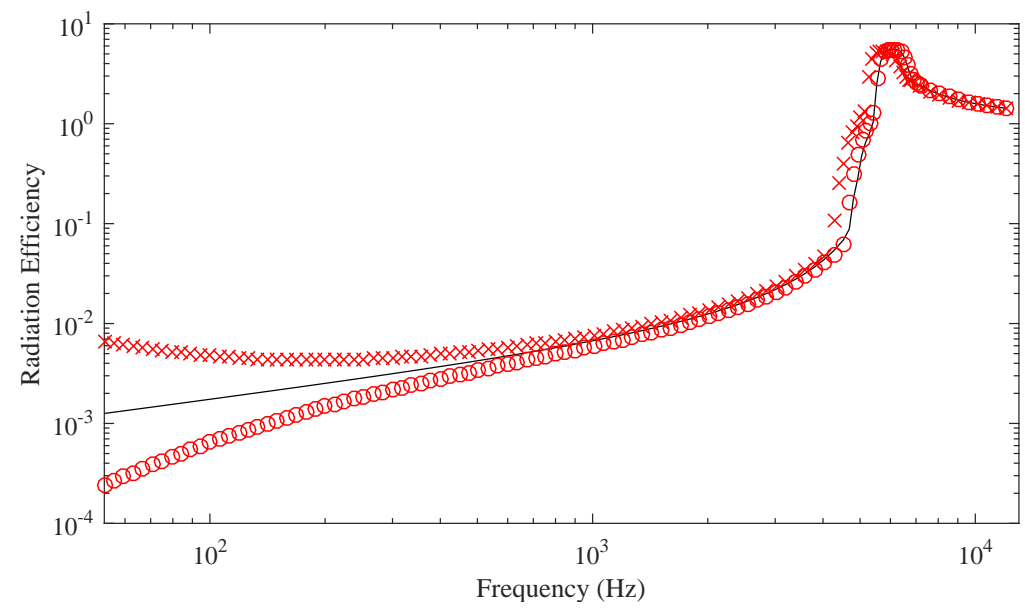

Figure 7: Comparison of the radiation efficiency of the monolithic laminate: (-) non-stressed, (x) pre-stressed, tensile force $\mathrm{T}=100 \mathrm{~N}$ in $x$ direction, (o) pre-stressed, compressive force $\mathrm{T}=-$ $100 \mathrm{~N}$ in $x$ direction 


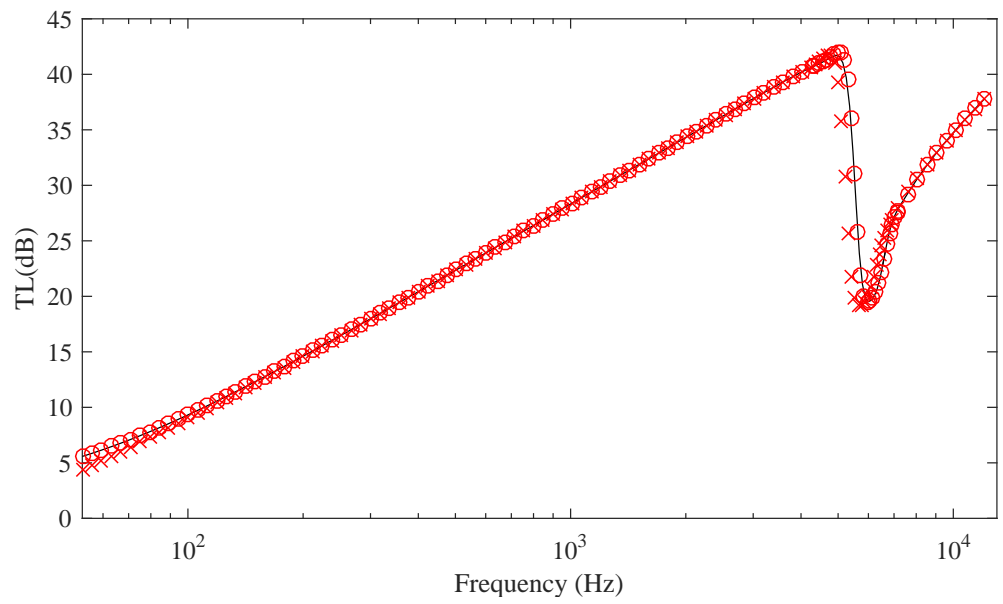

Figure 8: Comparison of the transmission loss of the monolithic laminate: (-) non-stressed, (x) pre-stressed, tensile force $\mathrm{T}=100 \mathrm{~N}$ in $x$ direction, (o) pre-stressed, compressive force $\mathrm{T}=-100 \mathrm{~N}$ in $x$ direction 


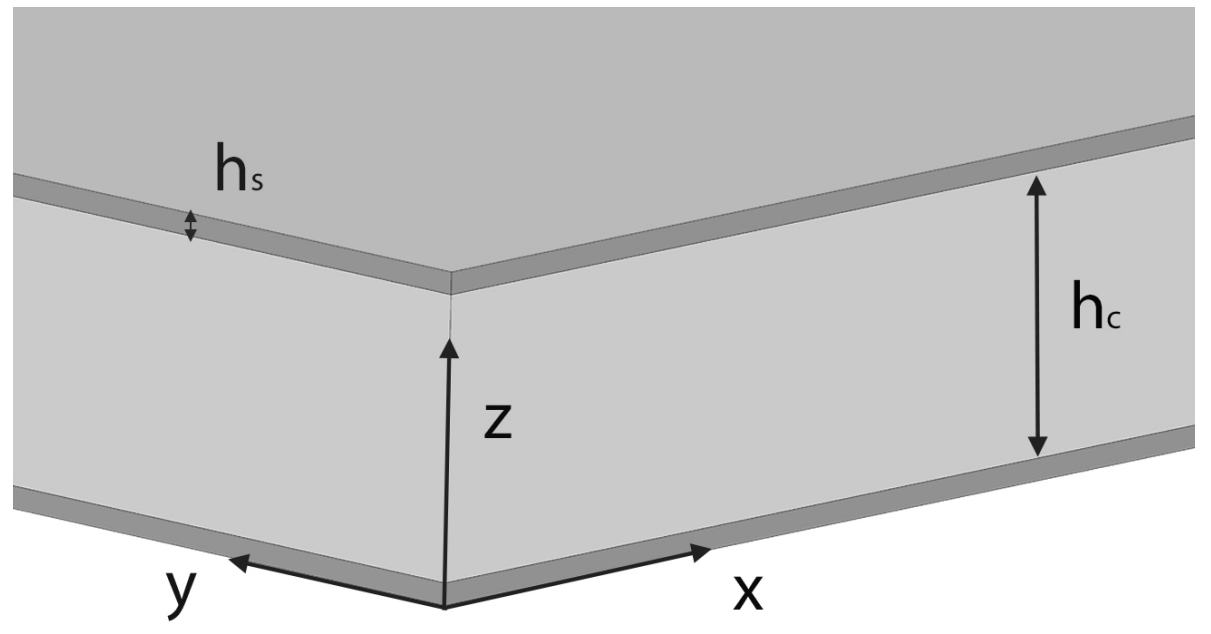

Figure 9: Representation of the sandwich laminate 


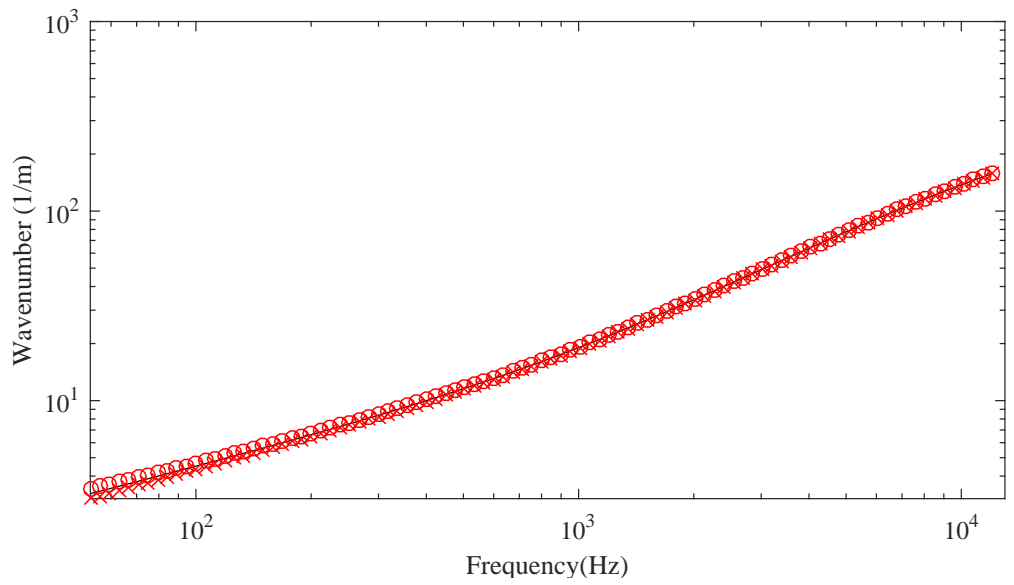

Figure 10: Comparison of the bending wavenumber (x-direction) of the sandwich plate: (-) non-stressed, (x) pre-stressed, tensile force $\mathrm{T}=100 \mathrm{~N}$ in $x$ direction, (o) pre-stressed, compressive force $\mathrm{T}=-100 \mathrm{~N}$ in $x$ direction 


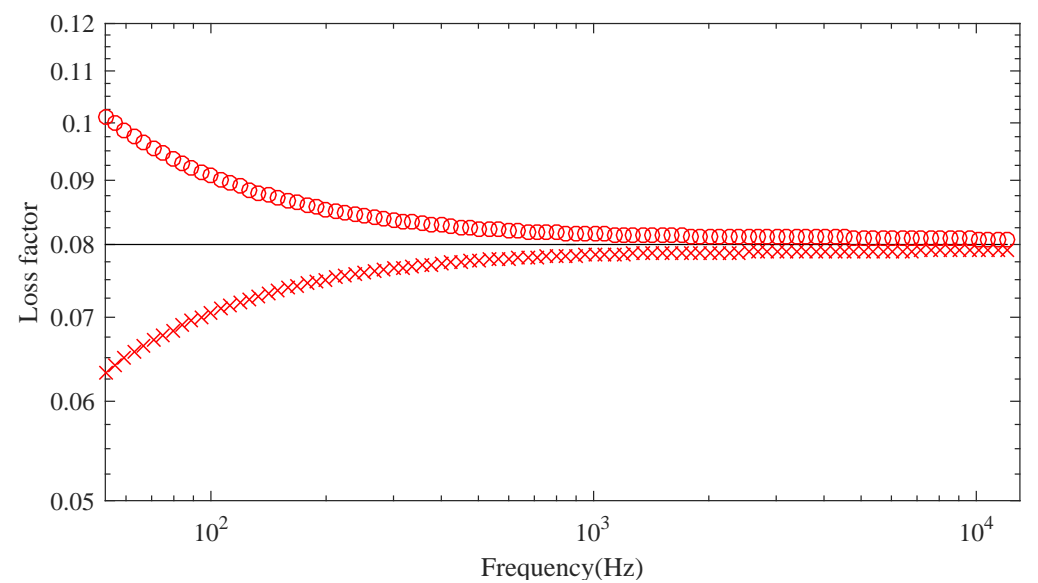

Figure 11: Comparison of the loss factor of the sandwich plate: (-) non-stressed, (x) prestressed, tensile force $\mathrm{T}=100 \mathrm{~N}$ in $x$ direction, (o) pre-stressed, compressive force $\mathrm{T}=-100 \mathrm{~N}$ in $x$ direction 


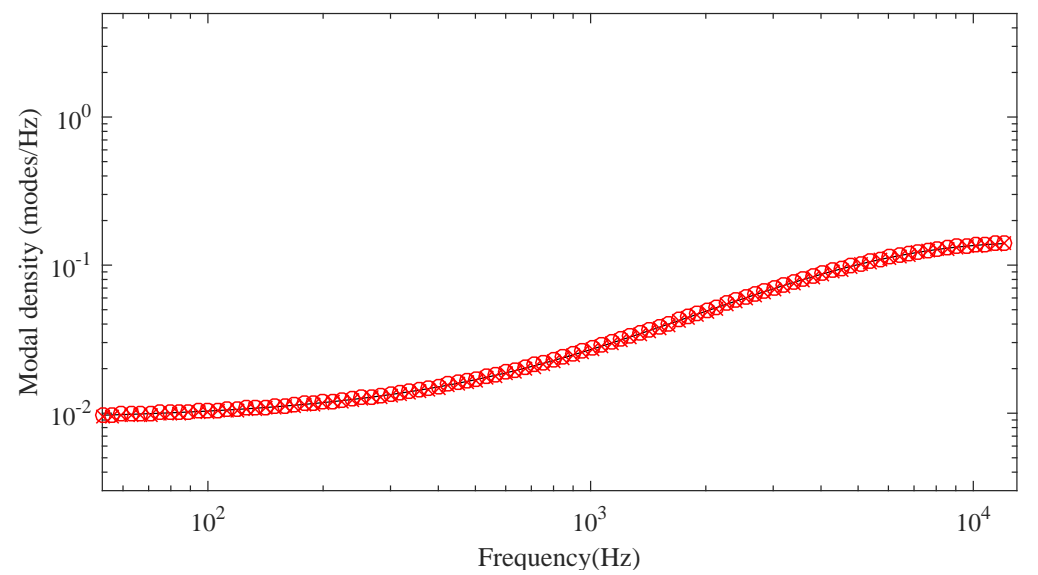

Figure 12: Comparison of the modal density of the sandwich plate: (-) non-stressed, (x) prestressed, tensile force $\mathrm{T}=100 \mathrm{~N}$ in $x$ direction, (o) pre-stressed, compressive force $\mathrm{T}=-100 \mathrm{~N}$ in $x$ direction 


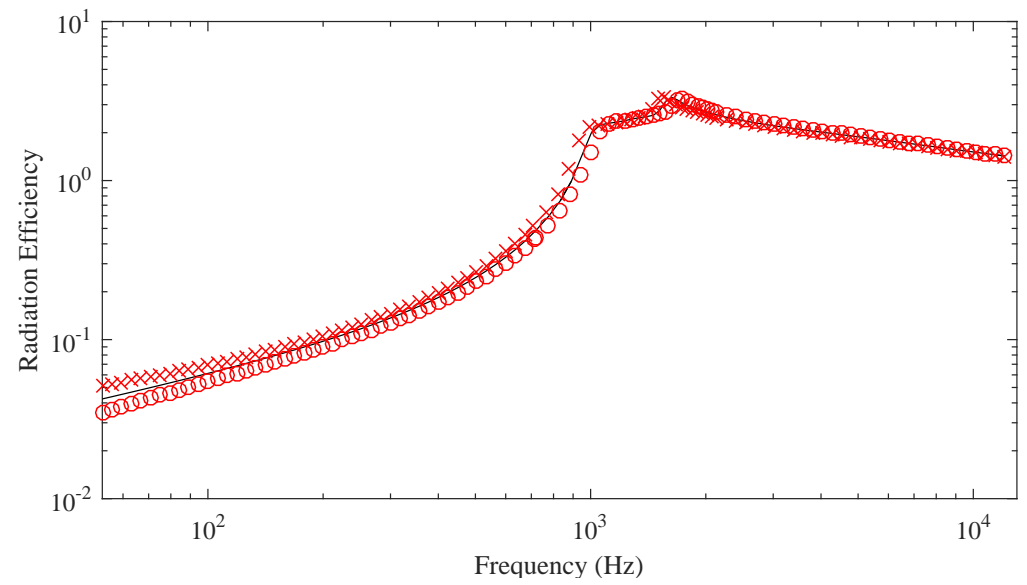

Figure 13: Comparison of the radiation efficiency of the sandwich plate: (-) non-stressed, (x) pre-stressed, tensile force $\mathrm{T}=100 \mathrm{~N}$ in $x$ direction, (o) pre-stressed, compressive force $\mathrm{T}=-100 \mathrm{~N}$ in $x$ direction 


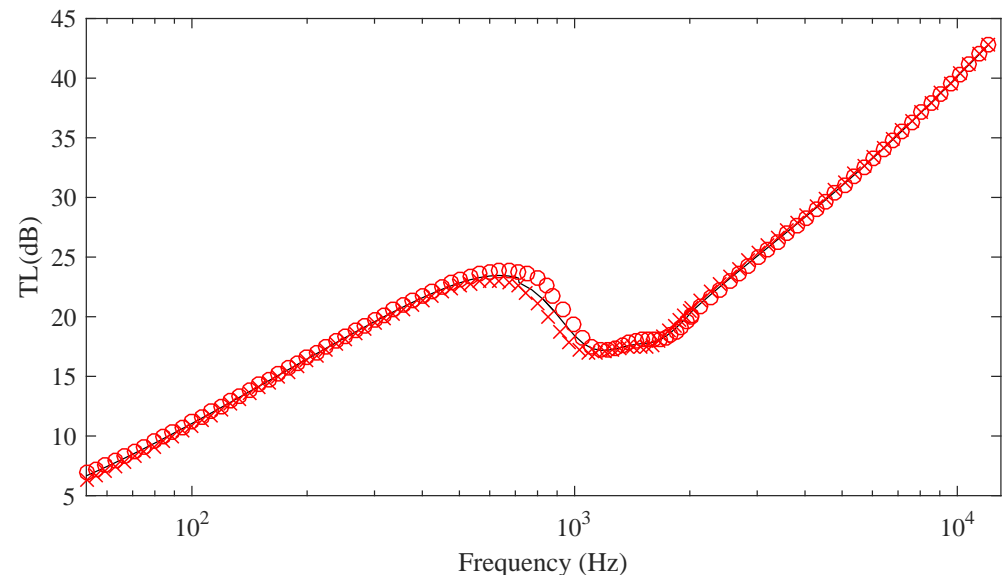

Figure 14: Comparison of the transmission loss of the sandwich plate: (-) non-stressed, (x) pre-stressed, tensile force $\mathrm{T}=100 \mathrm{~N}$ in $x$ direction, (o) pre-stressed, compressive force $\mathrm{T}=-100 \mathrm{~N}$ in $x$ direction 


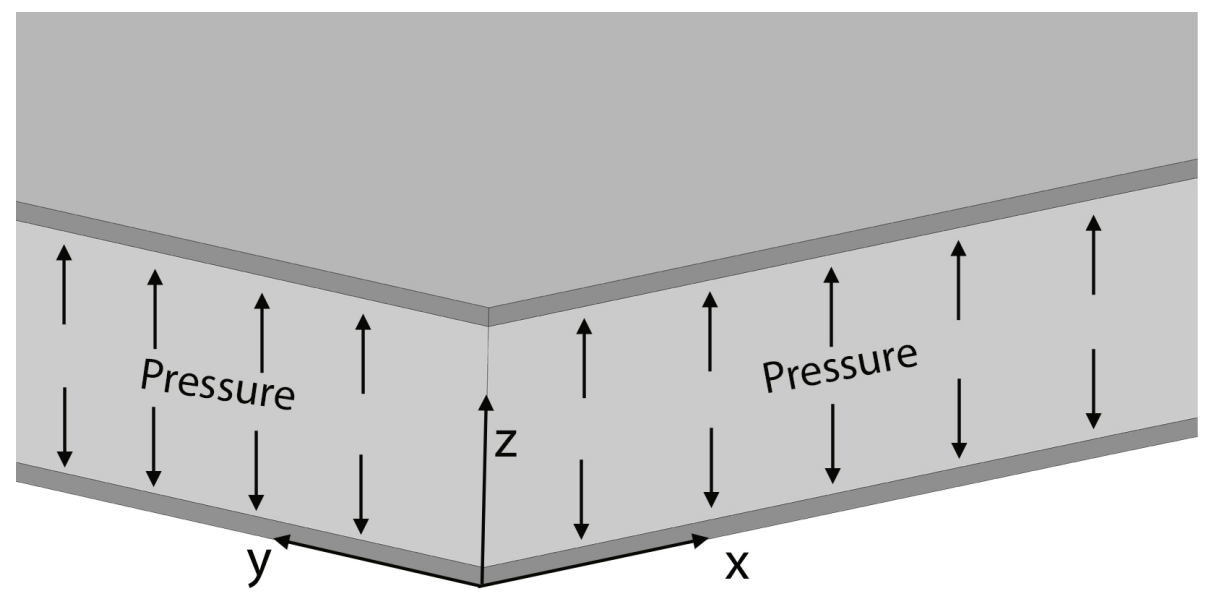

Figure 15: Representation of the internally pressurised sandwich laminate 


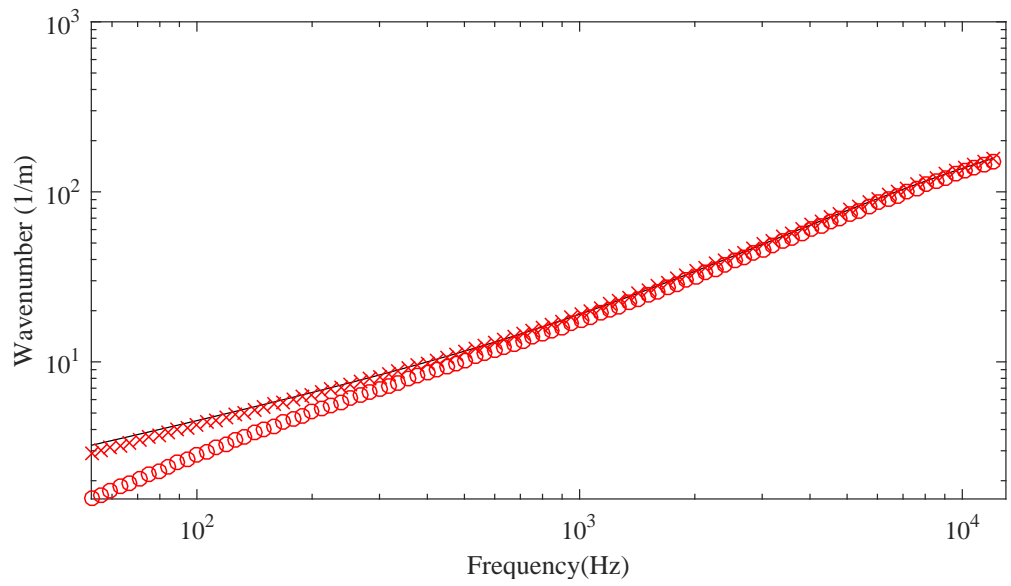

Figure 16: Comparison of the bending wavenumber (x-direction) of the sandwich plate: (-) non-pressurised, (x) pre-stressed, internal pressure $\mathrm{p}=10 \mathrm{bar},(\mathrm{o})$ pre-stressed, internal pressure $\mathrm{p}=100 \mathrm{bar}$ 


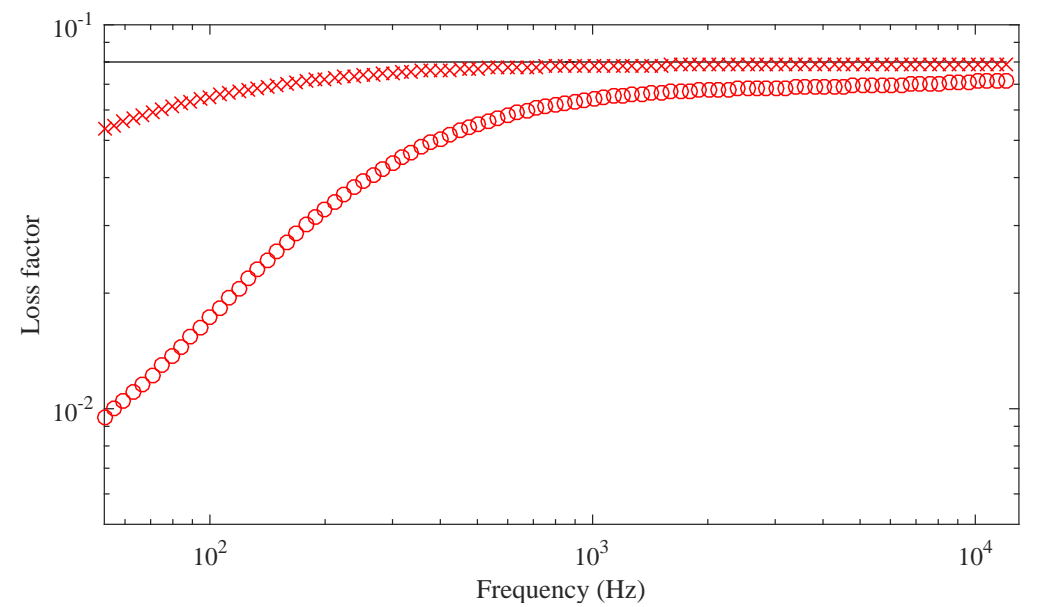

Figure 17: Comparison of the loss factor of the sandwich plate: $(-)$ non-pressurised, $(\mathrm{x})$ prestressed, internal pressure $\mathrm{p}=10 \mathrm{bar}$, $(\mathrm{o})$ pre-stressed, internal pressure $\mathrm{p}=100 \mathrm{bar}$ 


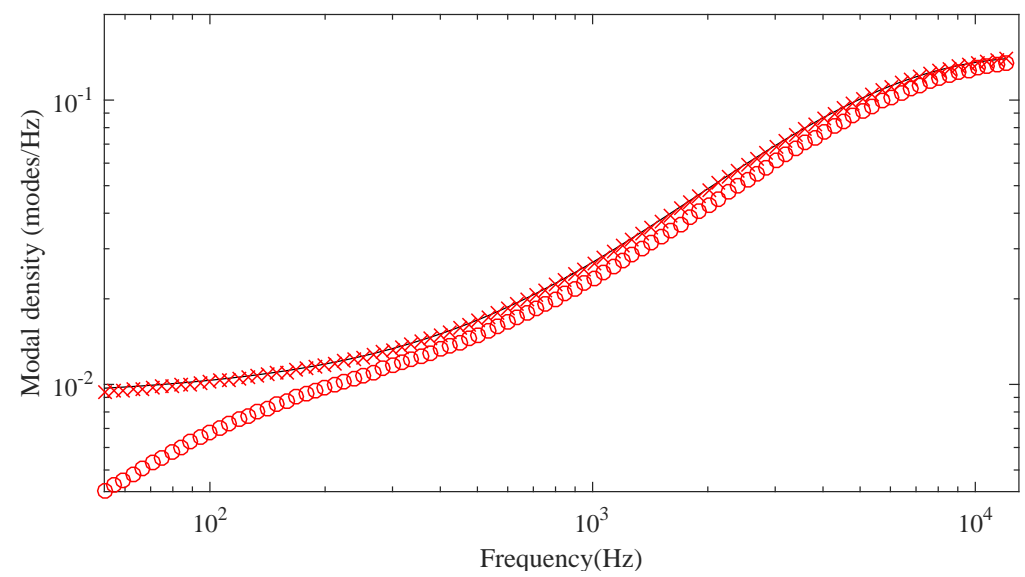

Figure 18: Comparison of the modal density of the sandwich plate: (-) non-pressurised, (x) pre-stressed, internal pressure $\mathrm{p}=10 \mathrm{bar},(\mathrm{o})$ pre-stressed, internal pressure $\mathrm{p}=100 \mathrm{bar}$ 


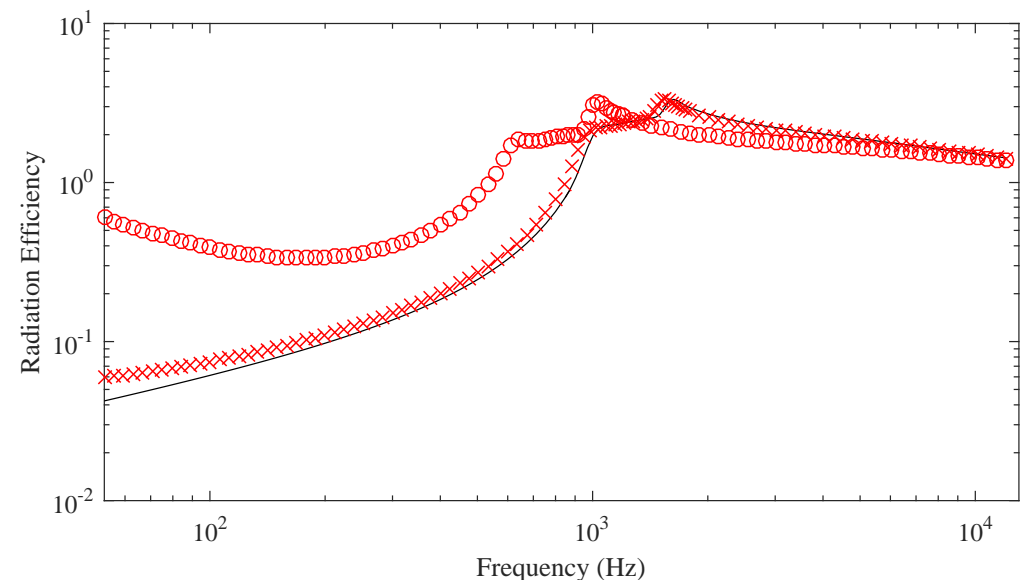

Figure 19: Comparison of the radiation efficiency of the sandwich plate: (-) non-pressurised, (x) pre-stressed, internal pressure $\mathrm{p}=10 \mathrm{bar}$, (o) pre-stressed, internal pressure $\mathrm{p}=100 \mathrm{bar}$ 


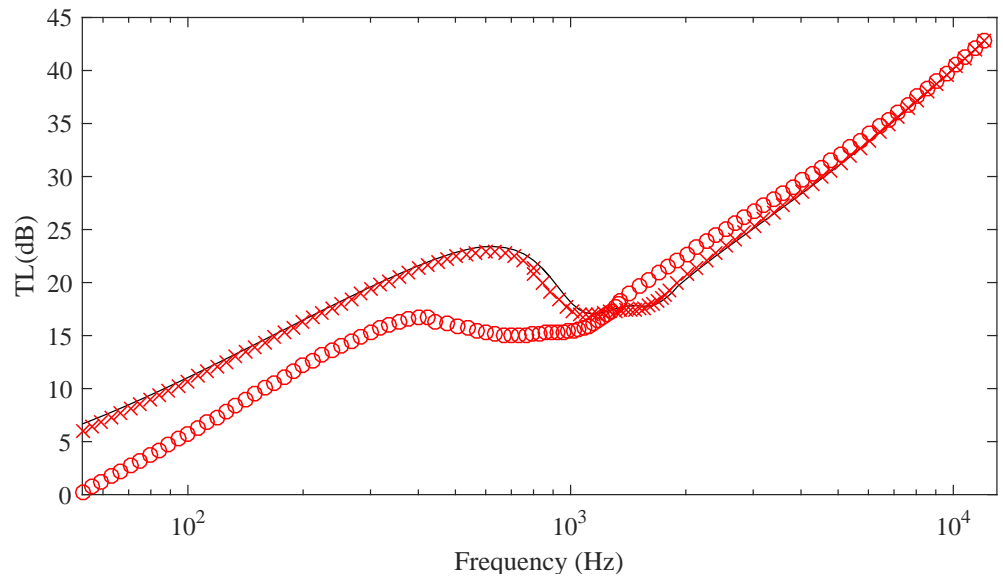

Figure 20: Comparison of the transmission loss of the sandwich plate: (-) non-pressurised, (x) pre-stressed, internal pressure $\mathrm{p}=10 \mathrm{bar},(\mathrm{o})$ pre-stressed, internal pressure $\mathrm{p}=100 \mathrm{bar}$ 


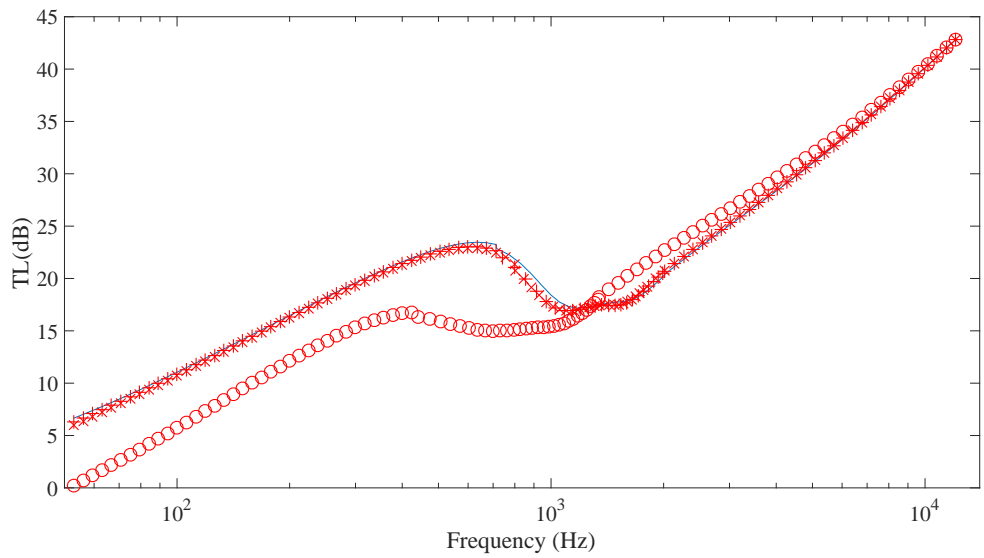

Figure 21: Comparison of the transmission loss of the sandwich plate: (-) non-stressed, (x) pre-stressed, internal pressure $\mathrm{p}=10 \mathrm{bar},(\mathrm{o})$ pre-stressed, internal pressure $\mathrm{p}=100 \mathrm{bar},(+)$ pre-stressed, tensile force $\mathrm{T}=100 \mathrm{~N}$ 


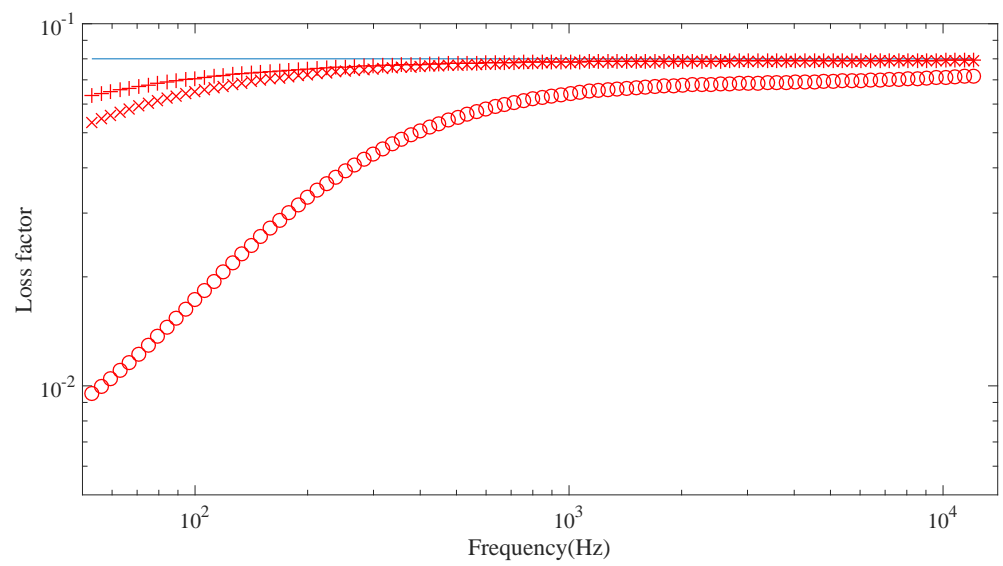

Figure 22: Comparison of the transmission loss of the sandwich plate: (-) non-stressed, (x) pre-stressed, internal pressure $\mathrm{p}=10 \mathrm{bar},(\mathrm{o})$ pre-stressed, internal pressure $\mathrm{p}=100 \mathrm{bar},(+)$ pre-stressed, tensile force $\mathrm{T}=100 \mathrm{~N}$ 


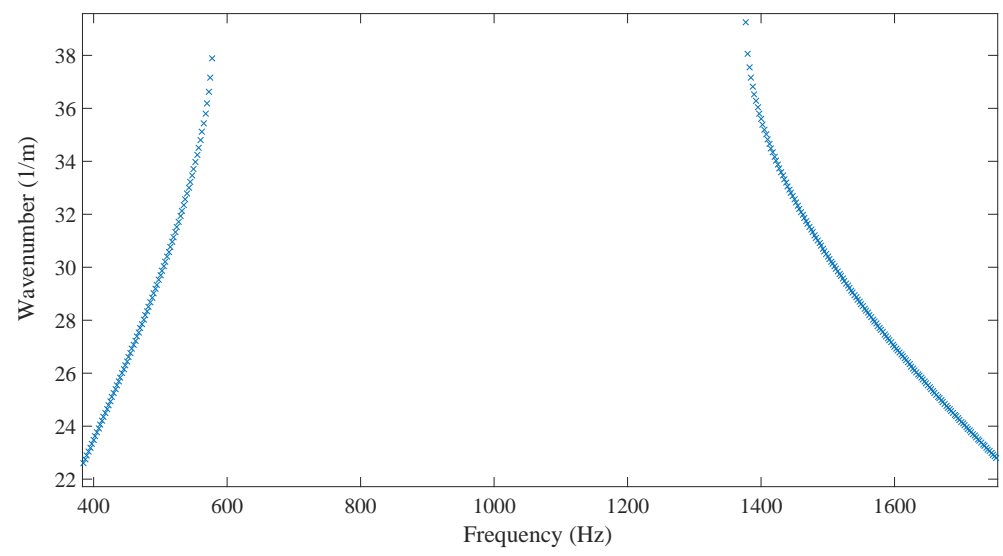

Figure 23: Wave dispersion graph of periodically pressured beam 


\section{List of Tables}

1 Material properties . . . . . . . . . . . . . . 49 
Table 1: Material properties

\begin{tabular}{ccc}
\hline \hline Material I & Material II & Material III \\
\hline$\rho=1870 \mathrm{~kg} / \mathrm{m}^{3}$ & $\rho=1550 \mathrm{~kg} / \mathrm{m}^{3}$ & $\rho=110 \mathrm{~kg} / \mathrm{m}^{3}$ \\
$E_{x}=60 e 9 P a$ & $E_{x}=48 e 9 P a$ & $E_{x}=145 e 6 P a$ \\
$E_{y}=60 e 9 P a$ & $E_{y}=48 e 9 P a$ & $E_{y}=145 e 6 P a$ \\
$E_{z}=40 e 9 P a$ & $E_{z}=48 e 9 P a$ & $E_{z}=145 e 6 P a$ \\
$\nu_{x y}=0.25$ & $\nu_{x y}=0.3$ & $\nu_{x y}=0.45$ \\
$\nu_{y z}=0.4$ & $\nu_{y z}=0.3$ & $\nu_{y z}=0.45$ \\
$\nu_{x z}=0.4$ & $\nu_{x z}=0.3$ & $\nu_{x z}=0.45$ \\
$G_{x y}=3.6 e 9 P a$ & $G_{x y}=2.8 e 9 P a$ & $G_{x y}=50 e 6 P a$ \\
$G_{y z}=1.2 e 9 P a$ & $G_{y z}=1.0 e 9 P a$ & $G_{y z}=50 e 6 P a$ \\
$G_{x z}=1.2 e 9 P a$ & $G_{x z}=1.0 e 9 P a$ & $G_{x z}=50 e 6 P a$ \\
\hline
\end{tabular}

